\title{
Development of powerful long-pulse Bragg FELs operating from sub-THz to THz bands based on linear induction accelerators: recent results and projects
}

\author{
N.Yu. Peskov $^{1}$, N.S. Ginzburg ${ }^{1}$, A.M. Malkin ${ }^{1}$, A.S. Sergeev ${ }^{1}$, V.Yu. Zaslavsky ${ }^{1}$,
} A.K. Kaminsky $^{2}$, S.N. Sedykh $^{2}$, I.I. Golubev ${ }^{2}$, S.M. Golubykh ${ }^{2}$, A.P. Kozlov ${ }^{2}$, A.I. Sidorov ${ }^{2}$, A.V. Arzhannikov ${ }^{3}$, D.A. Nikiforov ${ }^{3}$, S.L. Sinitsky ${ }^{3}$, D.I. Skovorodin ${ }^{3}$ and A.A. Starostenko ${ }^{3}$

${ }^{1}$ Institute of Applied Physics RAS, Nizhny Novgorod, Russia, peskov@appl.sci-nnov.ru

${ }^{2}$ Joint Institute for Nuclear Research, Dubna, Russia,

${ }^{3}$ Budker Institute of Nuclear Physics RAS, Novosibirsk, Russia

\section{Introduction}

To date, a series of successful experiments on the realization of powerful high-efficiency free-electron masers (FEMs) has been carried out in collaboration between JINR (Dubna) and IAP RAS (N.Novgorod) based on linac LIU-3000 0.8 MeV/200 A / 250 ns. The use of high-selective Bragg resonators of new types allowed stable regime of narrow-band generation to be obtained in these experiments up to W-band at multi-MW powers $[1,2]$. The radiation parameters achieved allows JINR-IAP FEM to be used in several applications including testing of components for highgradient accelerators, biology-medical studies, physics of nanoparticles, etc [3].

The aim of present work is advance of powerful long-pulse FELs into the $\mathrm{THz}$ frequencies. Such project is initiated based on the linear induction accelerators, which are developing in the BINP RAS (Novosibirsk) $5-20 \mathrm{MeV} / 2 \mathrm{kA} / 200 \mathrm{~ns}$. In this paper, we discuss the basic parameters of such FELs and the results of their computer simulations. As a microwave system capable to provide stable narrow-band operation in strongly oversize interaction space in these FELs, we consider so-called advanced Bragg structures with the feedback loop involving propagating and quasi-cutoff waves (Fig. 1) $[4,5]$.

\section{JINR-IAP FEM operating from Ka- to W-band}

The JINR-IAP FEM is driven by induction linac LIU-3000 (JINR). A reversed guide magnetic field configuration is used in the FEM, which possesses low sensitivity to the initial beam spread and, as a result, provides high-efficiency interaction. For operation from Ka- to $\mathrm{W}$-bands, helical wigglers having periods from $6 \mathrm{~cm}$ to $3 \mathrm{~cm}$ correspondingly were constructed. Enhance in amplitude of the transverse magnetic field in short-period wigglers alongside with refining its transverse homogeneity was achieved by optimization of the currents distribution in the wiggler winding. To improve the quality of helical electron beam formation at short wavelengths, the slowly uptapered wiggler entrance was optimized as well [2].

For operation in different parts of the millimeter wavelength band a series of combined two-mirror Bragg resonators, consisted from the up-stream highselective advanced Bragg reflector and down-stream weakly-reflecting conventional Bragg reflector (Fig.1a), was elaborated. Resonators of such type

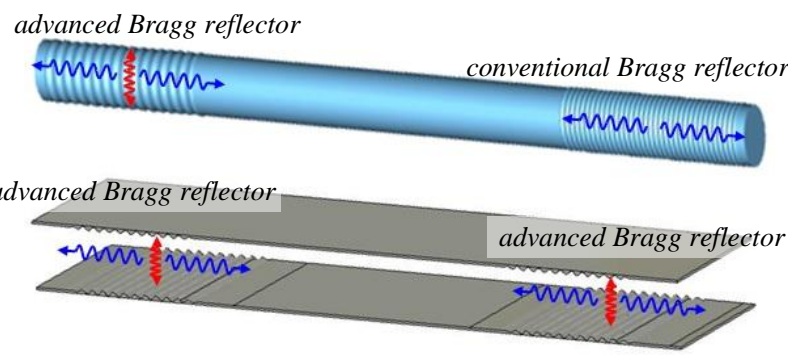

Fig. 1. Different schemes of Bragg resonators: (a) combined two-mirror resonator based on advanced and conventional Bragg reflectors of cylindrical geometry and (b) two-mirror resonator consisted from two advanced Bragg reflectors of planar geometry. The partial wave-fluxes in both reflectors are shown by the arrows.

allow decrease in Ohmic losses associated with excitation of the cut-off mode. For operation at $30 \mathrm{GHz}$, combined Bragg resonator was constructed with the oversized parameter $\varnothing / \lambda \sim 2$ and included advanced Bragg reflector with a feedback loop formed by two counter-propagating $\mathrm{TE}_{1,1}$ waves and cutoff $\mathrm{TE}_{1,2}$ wave. Resonator for $\mathrm{V}$-band $(60 \mathrm{GHz})$ was composed with advanced up-stream Bragg reflector having feedback loop $\mathrm{TE}_{1,1} \leftrightarrow \mathrm{TM}_{1,2}$ - cutoff $\leftrightarrow \mathrm{TE}_{1,1}$ at the oversize parameter $\varnothing / \lambda \sim 3$. For $\mathrm{W}$-band $(80 \mathrm{GHz})$ advanced Bragg reflectors based on excitation of the feedback cutoff wave of $\mathrm{TE}_{1,5}$ - type were designed to provide an effective FEM operation at oversize parameter $\emptyset / \lambda \sim 5$ (Fig. 2). In accordance with the 3D simulations, effective narrow-band reflections were demonstrated in "cold" test of novel Bragg structures. In all resonators described above the reflection band in advanced Bragg structures was measured 0.5 $0.7 \mathrm{GHz}$ with maximum power reflection up to 80 $90 \%$, while the conventional structures had $\sim 60 \%$ reflection in much broader band of $2.5-3 \mathrm{GHz}$.

In the proof-of-principle experiments at LIU3000 a narrow-band operation of novel scheme of FEM-oscillators was obtained under design parameters. At Ka-band, stable single-mode operation at the frequency of $30.2 \mathrm{GHz}$ was observed in accordance with simulations. The output power amounted up to 20 MW (efficiency about $15-20 \%$ ) with the spectrum width of $6-7 \mathrm{MHz}$ (measured by heterodyne technique) close to the theoretical limit (Fig. 3).

In the experiments at V- and $\mathrm{W}$-band, the radiation spectrum was measured by means of cut-off filters set with the accuracy of about $1 \mathrm{GHz}$. Both FEM demonstrated the oscillation frequency belonging to the designed feedback loop of the hybrid resonator in 

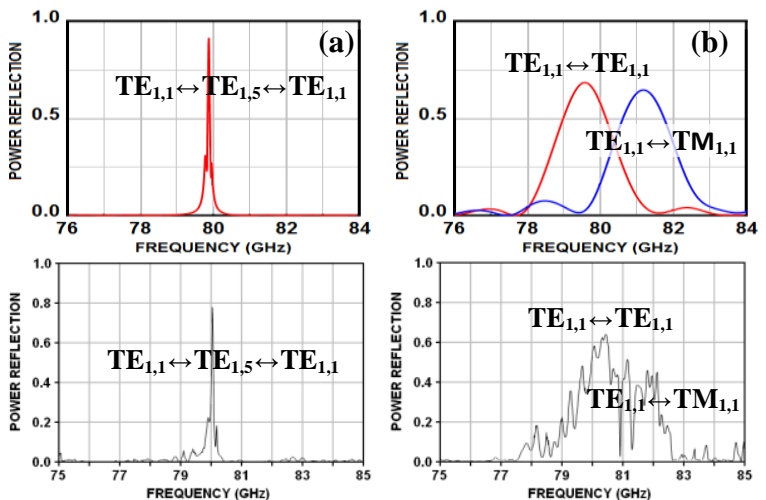

Fig. 2. Results of 3D simulations (top) and "cold" tests of Bragg structures of different types at W-band. Frequency dependence of reflection of (a) advanced $\left(l_{\mathrm{adv}}=12 \mathrm{~cm}\right)$ and (b) conventional $\left(l_{\text {con }}=8 \mathrm{~cm}\right)$ reflectors.

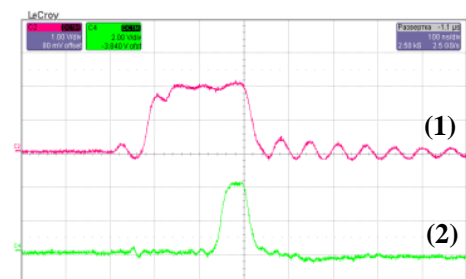

Fig. 3. Results of the JINR-IAP FEM experiments in Wband. Typical oscilloscope traces of (1) beam current and (2) output RF-pulse (100 ns / div.).

the vicinity of $59 \mathrm{GHz}$ and $80 \mathrm{GHz}$ correspondingly. In the resonators of optimal geometry the output power (measured by calorimeter) in both frequency regions amounted to 5 - $7 \mathrm{MW}$ when the beam current was about 70 - 100 A (Fig. 3), which corresponded to the electron efficiency of up to $10-12 \%$.

\section{Project of powerful Bragg FEL for THz-band}

Further increase of the radiation frequency in the JINR-IAP FEM is restricted by the wiggler period and electron beam energy. To advance FEL of this type into $\mathrm{THz}$ frequencies, it is attractive to exploit more powerful induction linac, which was elaborated currently at BINP RAS (the LIU-2 accelerator) [6]. This accelerator generates electron beam with the current of kA-level and energy of $2-5 \mathrm{MeV}$ (with the possibility to increase electrons energy up to $20 \mathrm{MeV}$ ).

Based on this beam, we initiated new project of multi-MW long-pulse THz FEL. In fact, the use of the beam with specified particles energy allows realization of the FEL in the range $1-10 \mathrm{THz}$ using the wiggler of $3-6 \mathrm{~cm}$ period. For the realized beam current of $\sim 1-2 \mathrm{kA}$, the radiation power is estimated on the level of $10-100 \mathrm{MW}$ even under the electron efficiency of $1-0.1 \%$ (which, obviously, would decrease with increase of the radiation frequency).

Results of simulation of the FEL driven by $5 \mathrm{MeV} / 1 \mathrm{kA}$ electron beam generated by the LIU - 2 are presented in Fig. 4. This beam is focused by the guide magnetic field of $\sim 0.15 \mathrm{~T}$ (in reversed configuration) and operating transverse electron velocities in the beam are pumped by the wiggler having $4 \mathrm{~cm}$ spatial period. For this FEL-oscillator we consider two-mirror resonator consisting of advanced Bragg reflectors of planar geometry (Fig.1b). Simulations demonstrated that Bragg structures of such type

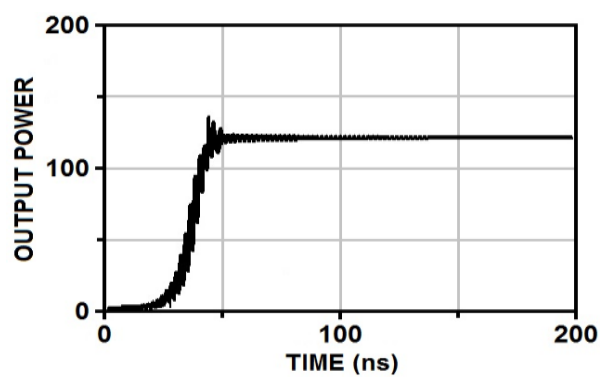

Fig. 4. Results of simulations of THz-band FEL based on linac LIU-2 (BINP RAS).

possess high reflectivity and ensure selectivity of the resonator for the transverse size (gap) up to 20 wavelengths. This size is sufficient for usage with the intense relativistic electron beams. For operation at $1 \mathrm{THz}$, we designed the resonator with $6 \mathrm{~mm}$ gap and the Bragg structures of $20 \mathrm{~cm}$ (up-stream) and $10 \mathrm{~cm}$ (down-stream) long having corrugation of the $0.3 \mathrm{~mm}$ period and $10-15 \mu \mathrm{m}$ depth, a regular section of 50 $70 \mathrm{~cm}$ long between them. According to the simulations, under design parameters the electron efficiency could achieve $2-3 \%$ and the output power reaches up to $50-100 \mathrm{MW}$. The Ohmic losses in this case do not exceed $25-30 \%$ from the radiated power.

Summarizing, novel conception of powerful $\mathrm{THz}-$ band Bragg FEL is developed. Original feature of this concept (in comparison with the others for now operating $\mathrm{THz}$ FEL) is the possibility to use electron beams with high-current (kA-level) and long-pulse (hundreds ns) duration. Such beams would allow achieving record levels of the radiation power of $10-100 \mathrm{MW}$ and pulse energy up to $10 \mathrm{~J}$ for the THz-band. Operability of key components of electrodynamic system for such FEL - advanced Bragg resonators - was demonstrated in the proof-ofprinciple experiments up to $\mathrm{W}$-band under oversized parameter $\varnothing / \lambda \sim 5$.

This work is partially supported by the Russian Foundation for Basic Research (grant \# 18-02-40009).

\section{References}

1. A.K. Kaminsky, E.A.Perelstein, S.N.Sedykh, e.a. Demonstration of powerful $30-\mathrm{GHz}$ FEM operation with resonant load // Tech. Phys. Lett. 2010. V.36 No.5 P.37.

2. N.Yu. Peskov, N.S. Ginzburg, A.K. Kaminsky, e.a. Powerful FEM-oscillators with advanced Bragg resonators operating in a single mode regime from Ka- to W-band // Proc. of the 41th Int. Conf. on IR, MM and THz Waves, Copenhagen, Denmark, 2016, p.F2E.01.

3. N.S. Ginzburg, I.I. Golubev, A.K. Kaminsky, e.a. Experiment on pulse heating and surface degradation of a copper cavity powered by powerful $30 \mathrm{GHz}$ free electron maser // Phys. Rev. Accel. Beams 2011. V.14. No.4. P.041002.

4. N.S. Ginzburg, A.M. Malkin, N.Yu. Peskov, e.a. Improving selectivity of free electron maser with 1D Bragg resonator using coupling of propagating and trapped waves // Phys. Rev. Accel. Beams 2005. V.8. P.040705.

5. N.S. Ginzburg, V.Y. Zaslavsky, I.V. Zotova, e.a. Terahertz Free-Electron Lasers with Bragg structures based on coupling between travelling and quasi-cutoff wases // JETP Lett. 2010. V.91. No.6. P.266.

6. P.V.Logachev, G.I.Kuznetsov, A A Korepanov, e.a. LIU-2 linear induction accelerator // Instruments and Experimental Techniques. 2013. V.56. No.6. P.672. 